\title{
A novel cascaded vector control scheme for the Modular Multilevel Matrix Converter
}

\author{
Felix Kammerer, Johannes Kolb and Michael Braun \\ Karlsruhe Institute of Technology (KIT) \\ Elektrotechnisches Institut (ETI) - Electrical Drives and Power Electronics \\ Kaiserstr. 12, 76131 Karlsruhe, Germany \\ felix.kammerer@kit.edu,www.eti.kit.edu
}

\begin{abstract}
This paper presents a novel cascaded vector control scheme for the Modular Multilevel Matrix Converter (M3C). The inner current control loops allow an independent control of the input and output converter currents. The outer energy control consists of average, vertical and horizontal balancing control loops to equalize the energy stored in the nine converter arms. A modulation method to balance the energy stored in the cells of one converter arm and to generate the desired arm voltage by selecting the appropriate cells is presented. The proposed vector control scheme allows the qualitative operation of the converter even under unbalanced line conditions. The function of the new converter control is verified by simulations. Additionally, three coupled three-phase $z$-winding arm inductors $L$ are presented for the use with the M3C.
\end{abstract}

\section{INTRODUCTION}

The Modular Multilevel Matrix Converter (M3C) shown in fig. 1 was presented in the year 2001 [1] without the arm inductors $L$. A space vector control method for the converter with one cell per converter arm which includes the balancing of the cell capacitors is explained in [2] and [3]. The presented control method is difficult to implement in M3Cs with more than one cell per arm, because the number of possible space vectors increases dramatically.

In [4] the M3C is presented with arm inductors. So the nine converter arms can work as controlled current sources. A feed forward control is implemented and simulation results show the steady state operation of the converter. In [5] a capacitor voltage balancing algorithm which generates DC circulating currents is added, but the proper function is not guaranteed under all circumstances. In [6], a cascaded feedback control system with nine inner arm current controllers and outer voltage controllers for the output voltage and the arm capacitor voltage is presented. Simulation results show the steady state operation of the converter, but the problem of balancing the cell capacitor voltages inside of one converter arm is not considered. Another drawback is that there are only eight degrees of freedom for the current control (the neutral points $N_{a}$ and $N_{e}$ are not connected), but they are using nine arm current controllers, for this reason the control system is overdetermined. In [7] the dimensioning of the $\mathrm{M} 3 \mathrm{C}$ and control principles based on the power balance are presented to feed gearless low-speed drives. The problem of balancing the energy is considered in case of an asymmetric grid or load.

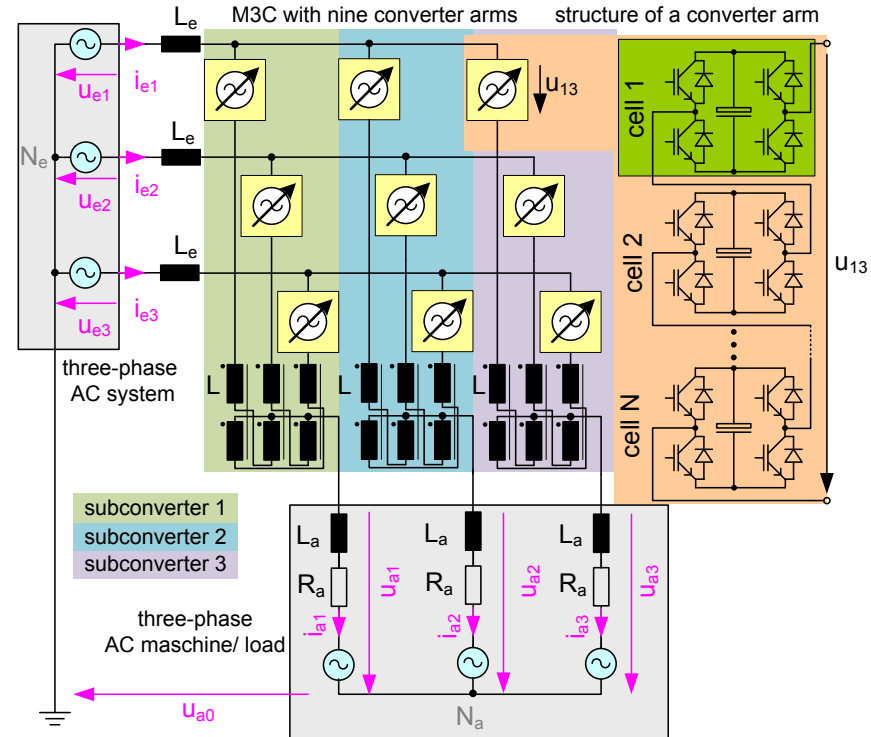

Fig. 1. Modular Multilevel Matrix Converter (M3C) with coupled three-phase Z-winding arm inductors $\mathrm{L}$

Up to now, it has not been worked out how to control the output currents independently from the input currents.

For this reasons it is necessary to implement a new control system for the M3C with the following characteristics:

- The inner current control needs eight current controllers, one for each degree of freedom. It is preferable to control the input and output converter currents independently instead of controlling the converter arm currents directly.

- Nine outer energy controllers are needed to keep the nine arm energies at a constant level (nine degrees of freedom). Controlling the mean energy and balancing the arm energies between the nine converter arms is preferred instead of controlling the nine arm energies directly.

- The problem of cell balancing inside of one converter arm needs to be considered in the control or modulation scheme without increasing the complexity of the system.

- The control systems must work stable even under unbalanced line conditions and dynamic changes of the operation point.

The M3C shown in fig. 1 consists of three subconverters. Each 


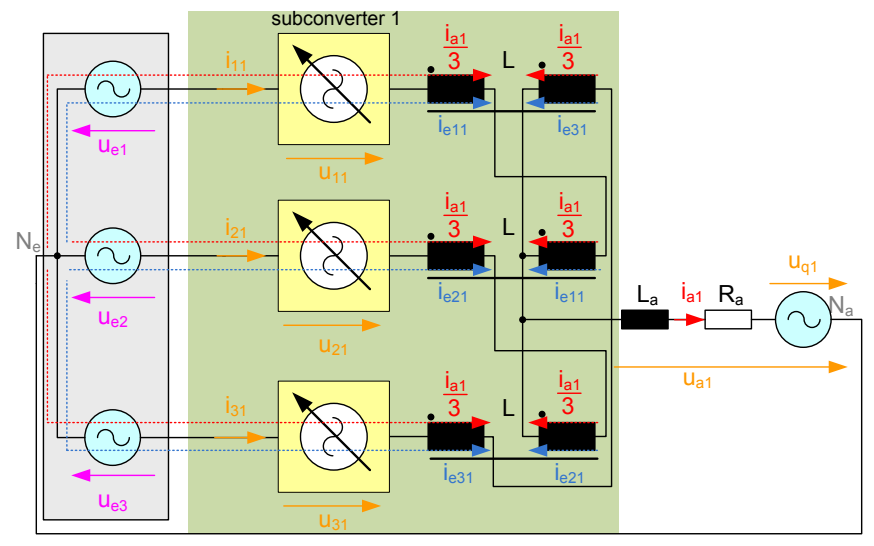

Fig. 2. Circuit diagram of one subconverter of the M3C with RL-Load and inner voltage $u_{q 1}$ and coupled three-phase $\mathrm{z}$-winding arm inductors $\mathrm{L}$

of them has the same structure as a star connected cascaded H-Bridge STATCOM with a load connected at the neutral point. [8] presents control principles for such a STATCOM using zero-sequence voltage and negative-sequence current for balancing the stored energy between the three converter arms. These control approaches are the basics for the balancing control of the $\mathrm{M} 3 \mathrm{C}$.

Additionally the independent arm inductors (see [4] and [7]) are replaced here with a coupled three-phase z-winding arm inductor $L$ for each of the three subconverters (fig. 1 and fig. 2). So, only the subconverter input currents become magnetically effective and the output currents are not affected by the inductor $L$. Because of this, only the input currents have to be considered for the dimensioning of the inductor core. So a smaller and for this reason cheaper inductor design with better electrical characteristics can be used to build the M3C. Note that the presented vector control scheme does not depend on these coupled inductors. It also works with independent arm inductors shown in [4] and [7].

\section{OPERATING PRINCIPALS OF THE M3C}

\section{A. Mathematical modeling of a subconverter}

For the mathematical modeling of the M3C it is helpful to connect the neutral points $N_{e}$ and $N_{a}$ temporarily for analyzing one subconverter independent of the two other subconverters. Fig. 2 shows the used circuit diagram of one subconverter, in which the line inductances $L_{e}$ are neglected. For symmetry reasons the approach that each converter arm must conduct one third of the output current $i_{a 1}$ and the corresponding subconverter input current is used:

$$
\begin{aligned}
i_{11} & =i_{e 11}+\frac{i_{a 1}}{3} \\
i_{21} & =i_{e 21}+\frac{i_{a 1}}{3} \\
i_{31} & =i_{e 31}+\frac{i_{a 1}}{3} \\
0 & =i_{e 11}+i_{e 21}+i_{e 31}
\end{aligned}
$$

The input and output currents are defined as:

$$
\begin{aligned}
i_{e 11} & =\hat{I}_{e 11} \cos \left(\omega_{e} t-\varphi_{e}\right) \\
i_{e 21} & =\hat{I}_{e 21} \cos \left(\omega_{e} t-\frac{2 \pi}{3}-\varphi_{e}\right) \\
i_{e 31} & =\hat{I}_{e 31} \cos \left(\omega_{e} t-\frac{4 \pi}{3}-\varphi_{e}\right) \\
i_{a 1} & =\hat{I}_{a} \cos \left(\omega_{a} t-\varphi_{a}\right)
\end{aligned}
$$

Next, the input and output currents can be described in $\alpha \beta 0$ components:

$$
\left[\begin{array}{c}
i_{e 1 \alpha} \\
i_{e 1 \beta} \\
i_{a 1}
\end{array}\right]=T_{1,3 \rightarrow \alpha \beta 0} \cdot\left[\begin{array}{c}
i_{11} \\
i_{21} \\
i_{31}
\end{array}\right]
$$

The transformation matrix $T_{1,3 \rightarrow \alpha \beta 0}$ is defined as:

$$
T_{1,3 \rightarrow \alpha \beta 0}=\left[\begin{array}{ccc}
\frac{2}{3} & -\frac{1}{3} & -\frac{1}{3} \\
0 & \frac{1}{\sqrt{3}} & -\frac{1}{\sqrt{3}} \\
1 & 1 & 1
\end{array}\right]
$$

From (3) follows that the output current $i_{a 1}$ is the zero sequence component of the arm currents $i_{11}, i_{21}$ and $i_{31}$. For this reason it is linear independent from the input current components $i_{e 1 \alpha}$ and $i_{e 1 \beta}$. The three outer voltage loops are defined as (see fig. 2):

$$
\begin{aligned}
& 0=-u_{e 1}+u_{11}+L \cdot \dot{i}_{11}+u_{a 1} \\
& 0=-u_{e 2}+u_{21}+L \cdot \dot{i}_{21}+u_{a 1} \\
& 0=-u_{e 3}+u_{31}+L \cdot \dot{i}_{31}+u_{a 1}
\end{aligned}
$$

With:

$$
u_{a 1}=R_{a} \cdot i_{a}+L_{a} \cdot \dot{i}_{a}+u_{q 1}
$$

$L$ is the effective arm inductance related to the input currents. Using (4) the $\alpha \beta 0$-components can be calculated:

$$
\begin{aligned}
& 0=-u_{e \alpha}+u_{1 \alpha}+L \cdot \dot{i}_{e 1 \alpha} \\
& 0=-u_{e \beta}+u_{1 \beta}+L \cdot \dot{i}_{e 1 \beta} \\
& 0=+3 u_{10}+3 u_{a 1}
\end{aligned}
$$

From (7) the arm voltages of subconverter one in $\alpha \beta 0$ components can be determinated:

$$
\begin{aligned}
& u_{1 \alpha}=u_{e \alpha}-L \cdot \dot{i}_{e 1 \alpha}=u_{e \alpha}-u_{L 1 \alpha} \\
& u_{1 \beta}=u_{e \beta}-L \cdot \dot{i}_{e 1 \beta}=u_{e \beta}-u_{L 1 \beta} \\
& u_{10}=-R_{a} \cdot i_{a}-L_{a} \cdot \dot{i}_{a 1}-u_{q 1}=-u_{a 1}
\end{aligned}
$$

Using the transformation matrix (10) the three arm voltages $u_{11}, u_{21}$ and $u_{21}$ which has to be generated by the subconverter are calulated as:

$$
\begin{aligned}
& u_{11}=u_{e 1}-u_{L 11}-u_{a 1} \\
& u_{21}=u_{e 2}-u_{L 21}-u_{a 1} \\
& u_{31}=u_{e 3}-u_{L 31}-u_{a 1}
\end{aligned}
$$

The transformation matrix $T_{1, \alpha \beta 0 \rightarrow 3}$ is defined as:

$$
T_{1, \alpha \beta 0 \rightarrow 3}=\left[\begin{array}{ccc}
1 & 0 & 1 \\
-\frac{1}{2} & \frac{\sqrt{3}}{2} & 1 \\
-\frac{1}{2} & -\frac{\sqrt{3}}{2} & 1
\end{array}\right]
$$


TABLE I

ACTIVE POWER COMPONENTS IN SUBCONVERTER 1

\begin{tabular}{c|c|c|c}
\hline Component & arm 11 & arm 21 & arm 31 \\
\hline input active power & $+\frac{1}{2} \hat{U}_{e} \cdot \hat{I}_{e} \cdot \cos \left(\varphi_{e}\right)$ & $+\frac{1}{2} \hat{U}_{e} \cdot \hat{I}_{e} \cdot \cos \left(\varphi_{e}\right)$ & $+\frac{1}{2} \hat{U}_{e} \cdot \hat{I}_{e} \cdot \cos \left(\varphi_{e}\right)$ \\
\hline output active power & $-\frac{1}{6} \hat{U}_{a} \cdot \hat{I}_{a} \cdot \cos \left(\varphi_{a}\right)$ & $-\frac{1}{6} \hat{U}_{a} \cdot \hat{I}_{a} \cdot \cos \left(\varphi_{a}\right)$ & $-\frac{1}{6} \hat{U}_{a} \cdot \hat{I}_{a} \cdot \cos \left(\varphi_{a}\right)$ \\
\hline additional active power for $f_{a}=0$ & $-\frac{1}{6} \hat{U}_{a} \cdot \hat{I}_{a} \cdot \cos \left(2 \gamma_{a}-\varphi_{a}\right)$ & $-\frac{1}{6} \hat{U}_{a} \cdot \hat{I}_{a} \cdot \cos \left(2 \gamma_{a}-\varphi_{a}\right)$ & $-\frac{1}{6} \hat{U}_{a} \cdot \hat{I}_{a} \cdot \cos \left(2 \gamma_{a}-\varphi_{a}\right)$ \\
\hline
\end{tabular}

$u_{L 11}, u_{L 21}$ and $u_{L 31}$ are the voltages to generate the desired input currents $i_{e 11}, i_{e 21}$ and $i_{e 31}$. Combining (9) and (5) and using (4) the derivations $\dot{i}_{e 1 \alpha}$ and $\dot{i}_{e 1 \beta}$ are given by:

$$
\begin{aligned}
& \dot{i}_{e 1 \alpha}=\frac{1}{L}\left(u_{L 1 \alpha}\right) \\
& \dot{i}_{e 1 \beta}=\frac{1}{L}\left(u_{L 1 \beta}\right)
\end{aligned}
$$

In the same way the derivation of the output current $\dot{i}_{a 1}$ is given by:

$$
\dot{i}_{a 1}=\frac{1}{L_{a}}\left(u_{a 1}-R_{a} i_{a 1}-u_{q 1}\right)
$$

So the input current controllers can be designed to control the input currents according to (11) and the output current controller according to (12). This result is also valid for the other two subconverters, so the output currents $i_{a 1}, i_{a 2}$ and $i_{a 3}$ can also be controlled in $\alpha \beta$-coordinates.

\section{B. Currents and voltages in one converter arm}

The minimum arm capacitor voltage (=sum of the capacitor voltages of all cells in one arm) to build the desired arm voltages (9) must fulfill the condition:

$$
u_{c, \min } \geq \hat{U}_{e, \max }+\hat{U}_{a, \max }+\hat{U}_{i e, \max }
$$

So the voltage transformation ratio $u=\frac{u_{a}}{u_{e}}$ of the input and output side depends only from the arm capacitor voltage. The maximum arm currents according to (1) can be calculated by:

$$
i_{a r m, \max } \geq \hat{I}_{e}+\frac{\hat{I}_{a}}{3}
$$

$\hat{I}_{e}$ and $\hat{I}_{a}$ are the amplitudes of the ideal input and output current systems of the subconverter.

\section{Energy balance of the $M 3 C$}

The nine converter arms of the $\mathrm{M} 3 \mathrm{C}$ consists of a series connection of $N$ cells. Each cell contains a H-bridge with an energy storage capacitor. The DC-capacitors of the cells do not have any power supply, comparable to the Modular Multilevel Converter (MMC) family presented in [9]. For this reason the arms can only deliver reactive power and the arm energy has to be controlled to keep the average value constant over the time. So the power balance of all nine converter arms has to be considered. During steady state operation of the M3C we

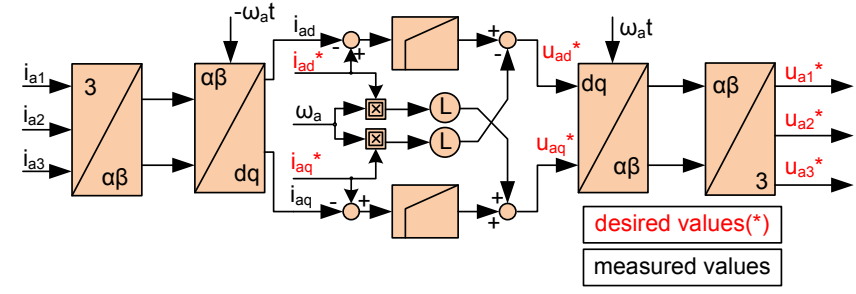

Fig. 3. Output current control of the M3C

get for the instantaneous power in arm 11:

$$
\begin{aligned}
p_{11}= & u_{11} \cdot i_{11} \\
= & {\left[+u_{e 1}-u_{a 1}\right] \cdot\left[+i_{e 11}+\frac{i_{a 1}}{3}\right] } \\
= & {\left[\hat{U}_{e} \cdot \cos \left(\omega_{e} t\right)-\hat{U}_{a} \cdot \cos \left(\omega_{a} t+\gamma\right)\right] } \\
& \cdot\left[\hat{I}_{e} \cdot \cos \left(\omega_{e} t-\varphi_{e}\right)+\frac{\hat{I}_{a}}{3} \cdot \cos \left(\omega_{a} t+\gamma-\varphi_{a}\right)\right]
\end{aligned}
$$

This is adopted for the three arms of subconverter 1. Table I shows the active power components for $f_{a} \neq f_{e}$. The reactive power terms which cause energy pulsation are neglected. This results in an active power balance even for zero output frequency $\left(f_{a}=0\right)$. In this case the active power supplied from the three subconverters is different and depends only on the angle $\gamma_{a}$ of the the output space vector. This does not affect the control and only has to be considered for the thermal design of the converter. For this reason the $\mathrm{M} 3 \mathrm{C}$ is suitable for high power operation at low output frequency. There is no need of internal balancing with circulation currents during steady state operation which is used in the MMC at low frequency operation [10]. The balancing currents reduce the maximum output power of the MMC at this operation point.

Therefore the $\mathrm{M} 3 \mathrm{C}$ is suitable to extend the MMC family for high power low frequency drive applications (see [7]). For example, the $\mathrm{M} 3 \mathrm{C}$ can replace cycloconverters which need a lot of filters for grid compliance.

\section{Vector Control Scheme FOR THE M3C}

\section{A. Output current control}

The three output currents $i_{a 1}, i_{a 2}$ and $i_{a 3}$ are transformed into $\alpha \beta$-coordinates and then rotated into stationary $d q$ coordinates. A pair of PI-controllers including feed-forward terms to eliminate the cross coupling are used (fig. 3). The output values are transformed back into phase output values. Subsequently they are used to build the desired arm voltages of the three subconverters (fig. 8). The desired values $i_{a d}^{*}$ and $i_{a q}^{*}$ 


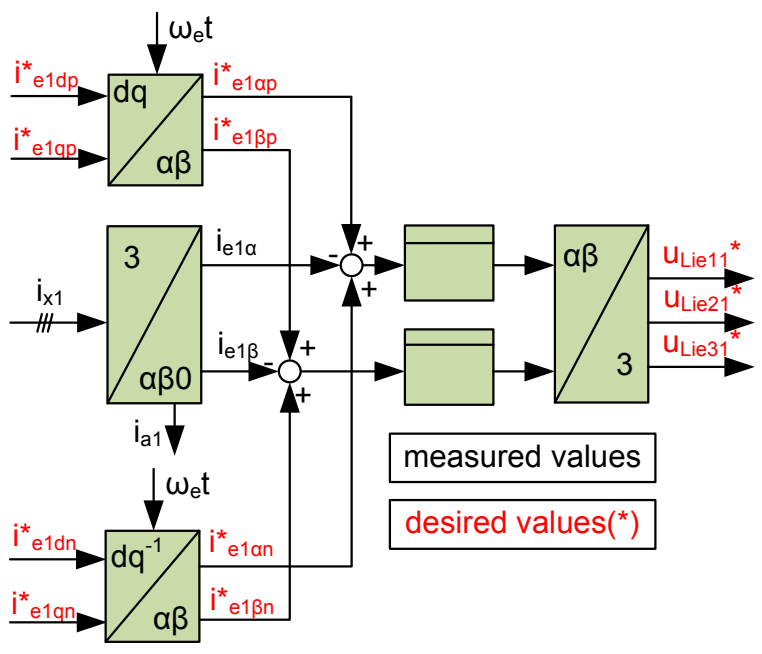

Fig. 4. Input current control of subconverter 1

are derived by a field or rotor oriented control of the connected three phase machine.

\section{B. Input current control of the subconverter}

The input currents are controlled independently for each subconverter in $\alpha \beta$-coordinates with a pair of proportional controllers (fig. 4). The desired positive sequence input dcurrent reference $i_{e 1 d p}^{*}$ is given by the outer energy and horizontal balancing control loop. The positive sequence input q-current reference $i_{e 1 q p}^{*}$ can be set to zero or to a desired value, for this reason the $\mathrm{M} 3 \mathrm{C}$ also has a STATCOM functionality. Both are rotated into $\alpha \beta$-coordinates using the following transformation matrix:

$$
\left[\begin{array}{l}
i_{e 1 \alpha p}^{*} \\
i_{e 1 \beta p}^{*}
\end{array}\right]=\left[\begin{array}{cc}
\cos \left(\omega_{e} t\right) & -\sin \left(\omega_{e} t\right) \\
\sin \left(\omega_{e} t\right) & \cos \left(\omega_{e} t\right)
\end{array}\right] \cdot\left[\begin{array}{l}
i_{e 1 d p}^{*} \\
i_{e 1 q p}^{*}
\end{array}\right]
$$

The negative sequence current references $i_{e 1 d n}^{*}$ and $i_{e 1 q n}^{*}$ are used for vertical balancing of the subconverter. They get their desired values from the vertical balancing control (fig. 7) and are rotated into $\alpha \beta$-coordinates using the following transformation matrix:

$$
\left[\begin{array}{l}
i_{e 1 \alpha n}^{*} \\
i_{e 1 \beta n}^{*}
\end{array}\right]=\left[\begin{array}{cc}
\cos \left(\omega_{e} t\right) & -\sin \left(\omega_{e} t\right) \\
-\sin \left(\omega_{e} t\right) & -\cos \left(\omega_{e} t\right)
\end{array}\right] \cdot\left[\begin{array}{l}
i_{e 1 d n}^{*} \\
i_{e 1 q n}^{*}
\end{array}\right]
$$

The output values are transformed back into the phase values to build the desired arm voltages of the three subconverters (fig. 8).

\section{Outer energy and balancing control}

First the nine arm capacitor voltages are transformed into an average value $u_{c 00}$ and eight unbalance components (fig. 5) using six times the following transformation matrix:

$$
\left[\begin{array}{l}
u_{c \alpha} \\
u_{c \beta} \\
u_{c 0}
\end{array}\right]=T_{2,3 \rightarrow \alpha \beta 0} \cdot\left[\begin{array}{l}
u_{c 1} \\
u_{c 2} \\
u_{c 3}
\end{array}\right]=\left[\begin{array}{ccc}
\frac{2}{3} & -\frac{1}{3} & -\frac{1}{3} \\
0 & \frac{1}{\sqrt{3}} & -\frac{1}{\sqrt{3}} \\
\frac{1}{3} & \frac{1}{3} & \frac{1}{3}
\end{array}\right] \cdot\left[\begin{array}{l}
u_{c 1} \\
u_{c 2} \\
u_{c 3}
\end{array}\right]
$$
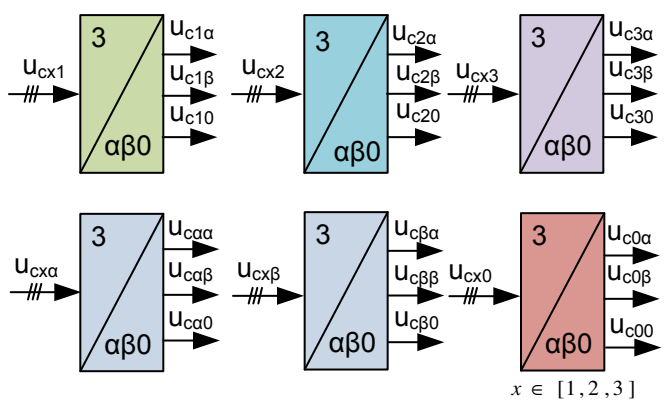

Fig. 5. Calculation of the average and unbalance arm capacitor voltages

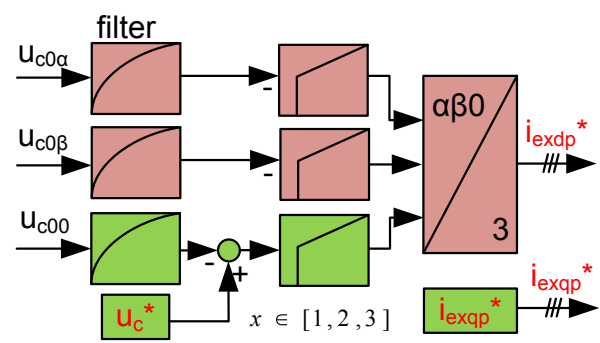

Fig. 6. Average energy and horizontal balancing control

The result contains the following eight different unbalance components:

- The values $u_{c 0 \alpha}$ and $u_{c 0 \beta}$ describe the horizontal unbalance of the arm capacitor voltages between the three subconverters.

- The values $u_{c \alpha \alpha}$ and $u_{c \alpha \beta}$ are used to control the vertical unbalance of the M3C in horizontal $\alpha$-direction.

- The values $u_{c \beta \alpha}$ and $u_{c \beta \beta}$ are used to control the vertical unbalance of the $\mathrm{M} 3 \mathrm{C}$ in horizontal $\beta$-direction.

- The values $u_{c \alpha 0}$ and $u_{c \beta 0}$ are used to control the zero sequence of the vertical unbalance of the M3C.

The advantage of this control concept is that the average control (fig. 6) of the arm energy has to control only DCcomponents during symmetrical operation of the converter. During balancing, only a small AC-component with line frequency $f_{e}$ has to be filtered, so a high dynamic and a good steady state operation behavior is guarantied with a PI-controller. For the horizontal balancing control only a frequency component of $2 f_{a}$ have to be filtered out and then $u_{c 0 \alpha}$ and $u_{c 0 \beta}$ has to be controlled to zero by distributing the positive sequence currents $i_{e 1 d p}^{*}, i_{e 2 d p}^{*}$ and $i_{e 3 d p}^{*}$ to their corresponding subconverters. For the vertical balancing control (fig. 7) a special method is used to ensure that the converter input currents $i_{e 1}, i_{e 2}$ and $i_{e 3}$ contain only positive sequence currents. The used methods with negative sequence currents and zero sequence output voltage are used to control STATCOMs (see [8]) and will be adapted here to use it together for the $\mathrm{M} 3 \mathrm{C}$ vertical balancing control. For this reason the vertical unbalance components of the three subconverters $u_{c 1 \alpha}, u_{c 1 \beta}, u_{c 2 \alpha}, u_{c 2 \beta}, u_{c 3 \alpha}$ and $u_{c 1 \beta}$ are transformed into $\alpha \beta 0$-components (fig. 5) and filtered afterwards. Here we have to filter out the frequencies $f_{e}, 2 f_{e}, f_{a}, 2 f_{a}$ and 


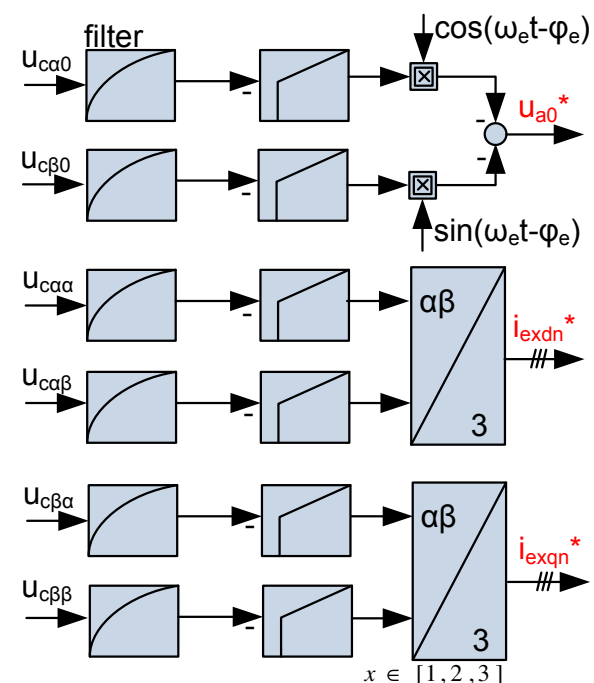

Fig. 7. Vertical balancing control of the three subconverters

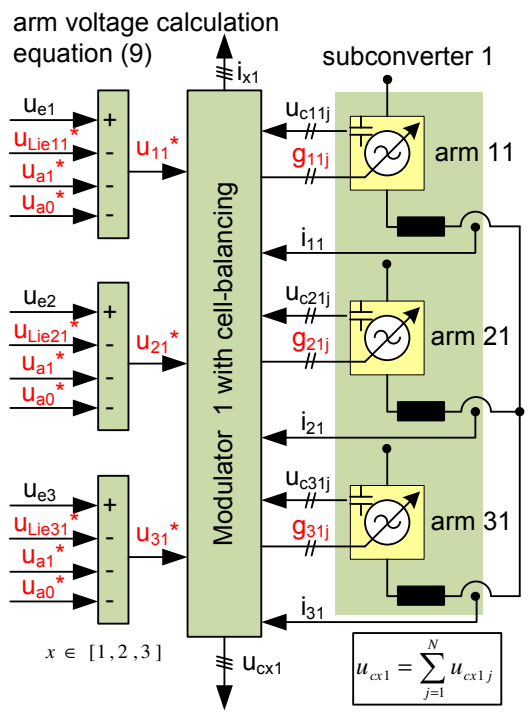

Fig. 8. Calculation of the arm voltages and modulation of subconverter 1

$f_{a} \pm f_{e}$. Due to this, the vertical balancing is slower than the horizontal balancing control. Then the four $\alpha \beta$-controllers are used to generate negative sequence currents which eliminate the horizontal difference of the vertical unbalance of the three subconverters. These currents are only internally used between the three subconverters and do not affect the input currents of the $\mathrm{M} 3 \mathrm{C}$. To control the zero sequence of the vertical unbalance a zero sequence output voltage $u_{a 0}$ with $f_{e}$ is generated. $u_{a 0}$ corresponds to the input currents and transfers active power between the three converter arms of the subconverters.

\section{Arm voltage calculation and modulation strategy}

Fig. 8 shows the arm voltage calculation with (9). The controller outputs are added to the measured line voltages to build the desired arm voltage. The modulator selects the

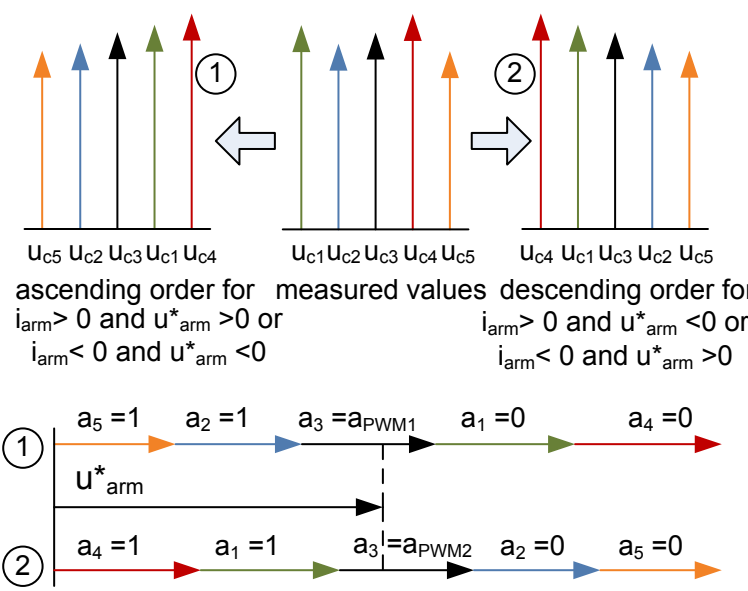

Fig. 9. Modulation and cell-balancing

cells which must be switched on and choses one cell which is PWM-modulated to build the desired voltage. Simultaneously the cell-balancing inside of one converter arm is realized. The procedure is based on the principals presented in [9] and similar to [10] and [11] except that an additional sorting condition which depends on the arm voltage polarity is added. Fig. 9 shows an example with $N=5$. Firstly the cell voltages are measured and then sorted ascending or descending depending of the arm power polarity. Secondly the necessary number of cells is switched on and one cell is modulated with the duty cycle $a_{P W M}$. Here for example $a_{P W M 1}$ and $a_{P W M 2}$ are calculated as:

$$
\begin{aligned}
& a_{P W M 1}=\frac{u_{a r m}^{*}-u_{c 5}-u_{c 2}}{u_{c 3}} \\
& a_{P W M 2}=\frac{u_{a r m}^{*}-u_{c 4}-u_{c 1}}{u_{c 3}}
\end{aligned}
$$

The other cells are switched off. With this sorting algorithm the cell-balancing is always ensured, because the cells with the lowest capacitor voltages are charged and the cells with the highest voltages are discharged.

\section{Simulation Results}

The proposed vector control scheme is implemented with one cell per arm $(\mathrm{N}=1)$ in MATLAB/Simulink and the SIMPOWER blockset to verify the function of the M3C. The parameters of the simulation are shown in table II. They are selected for the construction of a future laboratory prototype. Fig. 10 presents the simulation results. First the M3C operates with $\hat{i}_{a}=30 \mathrm{~A}$, which corresponds to an output power of $P_{a}=8,1 \mathrm{~kW}$. The output and input currents have a good sinusoidal waveform, but at the input side the ripple current caused by the switching of the cells is notable and depends on the number of cells $\mathrm{N}$ and also from the arm inductance $L$. For this reason the result will be better in a real M3C with more than one cell per arm. As expected the arm voltages and currents contain input and output frequency components. The arm capacitor voltages from subconverter 1 are maintained at 

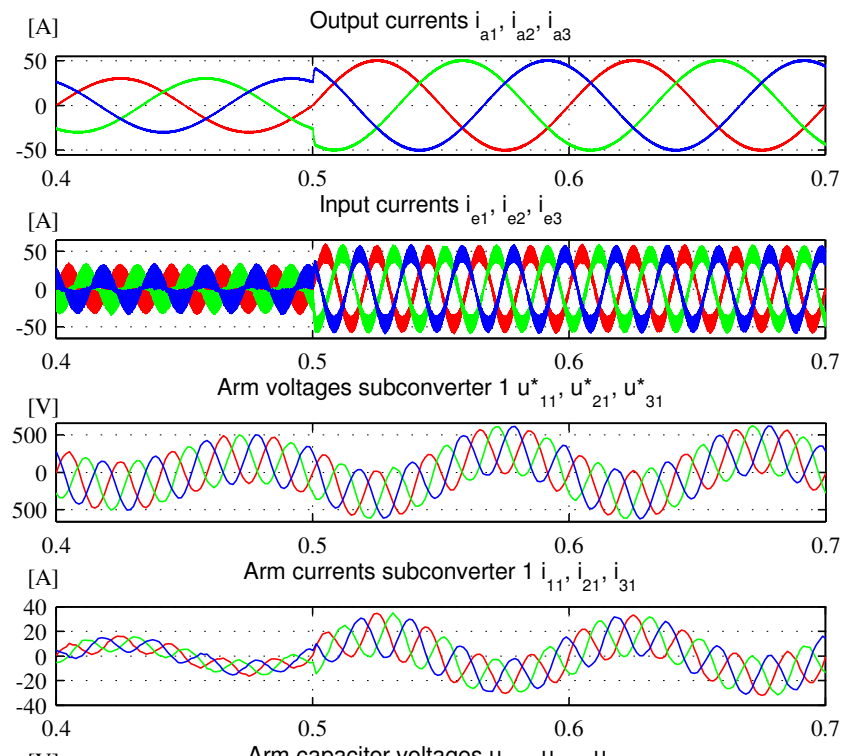

[V] Arm capacitor voltages $u_{c 11}, u_{c 21}, u_{c 31}$
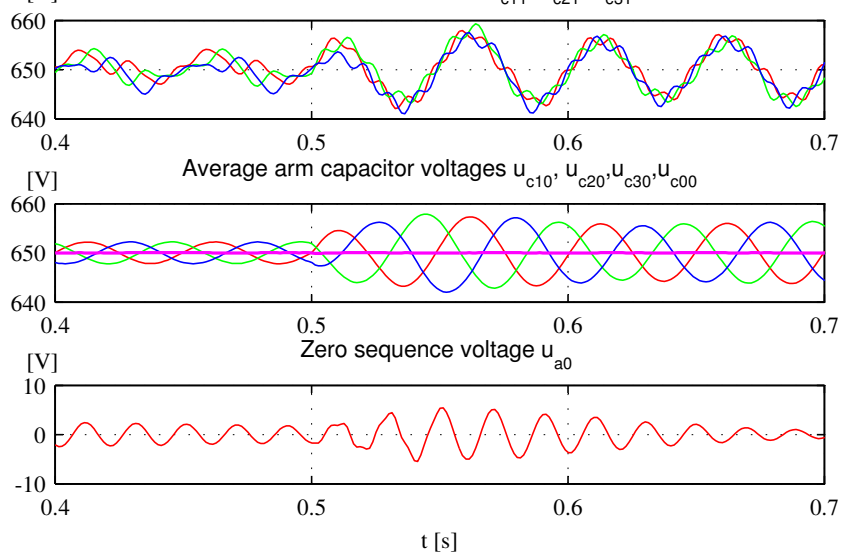

Fig. 10. Simulation results

their desired level. The average values of the three subconverters $u_{c 10}, u_{c 20}$ and $u_{c 30}$ contain only the frequency $2 f_{a}$ and a DC-component. Additionally the average value of the whole M3C $u_{c 00}$ which is almost a DC-component is shown. All nine arm capacitor voltages are maintained at the desired level by the energy and balancing control. The last picture shows the zero sequence voltage which is very low due to the symmetrical operation of the M3C. At $t=0.5 \mathrm{~s}$ the desired output current chances to $\hat{i}_{a}=50 \mathrm{~A}$, which corresponds to an output power of $P_{a}=22,5 \mathrm{~kW}$. Due to the higher load the energy pulsations in the converter arms increase. This has no negative effect to the M3C converter control which keeps the energy constant and balanced in the nine converter arms in about $0,15 \mathrm{~s}$. During balancing the zero sequence voltage $u_{a 0}$ increase temporally to distribute the energy between the converter arms.

\section{CONCLusion}

In this paper a novel cascaded vector control scheme for the $\mathrm{M} 3 \mathrm{C}$ with coupled three-phase $\mathrm{z}$-winding arm inductors $\mathrm{L}$ which consists of input and output current control loops as
TABLE II

SIMULATION PARAMETERS

\begin{tabular}{c|c}
\hline Parameter & Value \\
\hline sampling rate & $f_{t}=8 \mathrm{kHz}$ \\
\hline input voltage amplitude & $\hat{U}_{e}=325 \mathrm{~V}$ \\
\hline input frequency & $f_{e}=50 \mathrm{~Hz}$ \\
\hline arm capacitance & $C=5 \mathrm{mF}$ \\
\hline desired arm capacitor voltage & $U_{c}^{*}=650 \mathrm{~V}$ \\
\hline input inductance & $L_{e}=0 \mathrm{H}$ \\
\hline arm inductance & $L=800 \mu \mathrm{H}$ \\
\hline load inductance & $L_{a}=3 \mathrm{mH}$ \\
\hline load resistance & $R_{a}=6 \Omega$ \\
\hline inner voltage & $u_{q}=0 \mathrm{~V}$ \\
\hline output frequency & $f_{a}=10 \mathrm{~Hz}$ \\
\hline
\end{tabular}

well as outer energy and energy balancing control loops is presented. The complexity does not increase with the number of cells $N$ in one converter arm and makes the M3C scalable. An arm modulation method to balance the energy stored in the cells of one converter arm is added. With this control scheme the $\mathrm{M} 3 \mathrm{C}$ is suitable to work even under unbalanced line conditions and dynamic change of the operation point. It is easy to add a field or rotor oriented control to use the M3C for low frequency drive applications. The effectiveness of the robust control scheme for the inner energy balancing is demonstrated and verified by simulations.

\section{REFERENCES}

[1] R. Erickson and O. Al-Naseem, "A new family of matrix converters," in Industrial Electronics Society, 2001. IECON '01. The 27th Annual Conference of the IEEE, vol. 2, 2001, pp. 1515 -1520 vol.2.

[2] S. Angkititrakul and R. Erickson, "Control and implementation of a new modular matrix converter," in Applied Power Electronics Conference and Exposition, 2004. APEC 'O4. Nineteenth Annual IEEE, vol. 2, 2004, pp. $813-819$ vol. 2 .

[3] _ - "Capacitor voltage balancing control for a modular matrix converter," in Applied Power Electronics Conference and Exposition, 2006. APEC '06. Twenty-First Annual IEEE, march 2006, p. 7 pp.

[4] C. Oates, "A methodology for developing chainlink converters," in Power Electronics and Applications, 2009. EPE '09. 13th European Conference on, sept. 2009, pp. $1-10$.

[5] C. Oates and G. Mondal, "Dc circulating current for capacitor voltage balancing in modular multilevel matrix converter," EPE 2011 Birmingham, aug. 2011.

[6] D. C. Ludois, J. K. Reed, and G. Venkataramanan, "Hierarchical control of bridge-of-bridge multilevel power converters," Industrial Electronics, IEEE Transactions on, vol. 57, no. 8, pp. 2679 -2690, aug. 2010.

[7] A. Korn, M. Winkelnkemper, P. Steimer, and J. Kolar, "Direct modular multi-level converter for gearless low-speed drives," EPE 2011 Birmingham, aug. 2011.

[8] N. Hatano and T. Ise, "Control scheme of cascaded h-bridge statcom using zero-sequence voltage and negative-sequence current," Power Delivery, IEEE Transactions on, vol. 25, no. 2, pp. $543-550$, april 2010.

[9] M. Glinka and R. Marquardt, "A new ac/ac multilevel converter family," Industrial Electronics, IEEE Transactions on, vol. 52, no. 3, pp. $662-$ 669 , june 2005.

[10] J. Kolb, F. Kammerer, and M. Braun, "A novel control scheme for low frequency operation of the modular multilevel converter," PCIM Europe 2011, may 2011.

[11] S. Rohner, S. Bernet, M. Hiller, and R. Sommer, "Modulation, losses, and semiconductor requirements of modular multilevel converters," Industrial Electronics, IEEE Transactions on, vol. 57, no. 8, pp. 2633 -2642 , aug. 2010. 